\title{
Evolution of the interfacial shape in dissolutive wetting: Coupling of wetting and dissolution
}

\author{
Jinhong Yang, Quanzi Yuan*, Ya-Pu Zhao* \\ State Key Laboratory of Nonlinear Mechanics, Institute of Mechanics, Chinese Academy of Sciences, Beijing 100190, People's Republic of China \\ School of Engineering Science, University of Chinese Academy of Sciences, Beijing 100049, People's Republic of China
}

\section{A R T I C L E I N F O}

\section{Article history:}

Received 24 May 2017

Received in revised form 6 October 2017

Accepted 24 October 2017

Available online 3 November 2017

\section{Keywords:}

Dissolutive wetting

Droplet

Contact line

Moving interface

\begin{abstract}
A B S T R A C T
Interface evolution of a droplet on a dissolvable substrate is controlled by coupling of wetting and dissolution, which makes dissolutive wetting more complicated than wetting on inert substrate. In our experiments, xylene and polystyrene are used as solvent and solute, respectively. We record the continuous evolution of interfacial shape and measure change of geometrical parameters. Then, a model is built based on the Noyes-Whitney equation. The evolution mechanisms of interface and solute concentration under the combined actions of wetting and dissolution are analyzed. There are two stages in dissolutive wetting. The two stages are identified by the speed ratio of wetting and dissolution, which reflects the coupling between wetting and dissolution. The model can recover the evolution of the interfacial shape observed in the experiment. Our work may help to predict change of interfacial shape in dissolutive wetting, and the results provide a better understanding of dissolutive wetting which is a process that occurs in many important application areas.
\end{abstract}

(c) 2017 Elsevier Ltd. All rights reserved.

\section{Introduction}

Dissolutive wetting is a process in which a liquid wets and dissolves the substrate. It is a basic of reactive wetting. As for reactive wetting, the liquid have chemical reaction with the substrate by dissolution or producing reaction products at interface. The resulting reaction will strongly change the properties of interface, especially the contact line [1]. The early of technological interest in reactive wetting is focused on applications in joining processes between metals and ceramics [1,2]. However, dissolutive wetting is also ubiquitous and plays an important role in the fields of protection of optical components [3], digestion of fat [4] and steroids [5], printing [6], micro/nano-fluidics [7], fabrication of alloy nanowire [8,9], storage of carbon dioxide [10], and etc. In some applications, dissolutive wetting needs to be avoided. Even if some materials are durable such as Borosilicate glasses, there also exists very slow dissolutive wetting [11]. In some cases, dissolutive wetting is a much sought-after process. For example, etching that fabricates microstructures using silicon has been broadly applied

\footnotetext{
* Corresponding authors at: State Key Laboratory of Nonlinear Mechanics, Institute of Mechanics, Chinese Academy of Sciences, Beijing 100190, People's Republic of China. School of Engineering Science, University of Chinese Academy of Sciences, Beijing 100049, People's Republic of China.

E-mail addresses: yzhao@imech.ac.cn (Y.-P. Zhao), yuanquanzi@lnm.imech.ac.cn (Q. Z. Yuan).
}

in MEMS [12]. The design of drug also needs to take account of dissolutive wetting [13-16]. Predictions and control of the interfacial shape of dissolutive wetting in these applications are desirable. Not least motivated by these applications, the mechanisms that wetting interacts with dissolution come to attention of both chemistry and physics [17].

Compare with non-reactive wetting [18], dissolutive wetting involves many complex chemical and physical processes such as wetting, diffusion, and convection, which change components and their distributions in solvent. These processes change properties of solvent. Because of the complexity of the combined actions of wetting and dissolution, our fundamental understandings of dissolutive wetting mechanism relatively lag behind. For the theoretical investigations of dissolutive wetting, as far as we are concerned, there are three methods. One is thermodynamic method [19] For example, Yost et al. [20] used the early work of Onsager to calculate the evolution of contact line and contact angle. However, it was difficult to describe change of solid-liquid interface. The second method is fluid mechanics method. Warrant et al. [21] assumed solute was transported by diffusion primarily. However, they did not consider the influence of surface tension. The model of Braun et al. [22] was built according to lubrication theory. However, their model was so complex that the shape of interface could not be represented by a function. Alleborn et al. [23]. built a model to describe the interfacial shape near triple phase point in dissolutive wetting. The third method combines 
the N-S equations and thermodynamic theory. For example, Villanueva et al. [24] presented a multicomponent and multiphase model, and their model agreed with the experimental data successfully. However, the droplet was supposed to be thin and incompressible. In the above models, the surface tension of liquid was assumed as constant or brief function, and another important assumption was that the upper is spherical shape.

Pioneering studies also used simulated methods $[25,26]$ to calculate the evolution of boundaries especially the Arbitrary Lagrangian-Eulerian (ALE) method. This method have outstanding capability to deal with large deformation and capture the interface [27]. In experimental study, the Cu-Si system and Bi-Sn system were used commonly. Protsenko et al. [28] conducted experiments on $\mathrm{Cu}-\mathrm{Si}$ system to reveal the relation between weak concentration gradient and solid-liquid interface angle. Yin et al. [29] observed the initial concentration of liquid determined the speed of wetting. Previous experiments were conducted using opaque materials, so there were difficult to continuously record the process of interfacial evolution.

In this paper, we employed both experiment and theory to study the evolution of the interfacial shape in dissolutive wetting. In our studies, we use transparent xylene and polystyrene for experiments, measure the dissolutive wetting of small droplets on a dissolvable substrate, and discover the radius of droplet increases as $\mathrm{t}^{0.11}$. Based on Noyes-Whitney equation, we develop a model to describe change of interface and concentration of droplet. Our model can reproduce the results of experiment. Base on this model, we analyze the competition speeds of wetting between dissolution, which decides the dissolutive wetting in two stages. We hope that our theoretical solution for the wetting liquid dissolves the substrate will shed light on the coupling of wetting and dissolution in dissolutive wetting. Our work can be further applied to the design of products of MEMS and medicine field, and to the protection of key components in equipment.

\section{Experiment procedures}

\subsection{Dissolutive wetting experiments}

In the experiments, polystyrene and xylene are chosen to be solute and solvent, respectively. The polystyrene substrates are polished and cleaned by ultrasonic cleaning. Fluorescent agent is added to the xylene according to mass ratio of $800: 1$ to enhance the clarity of interface. The fluorescent agent will become green under the excitation of white light. The experimental process of dissolutive wetting is observed and recorded by using dark field mode of a microscope (KH-8700, Hirox). The relative influences
Table 1

The properties of solute and solvent.

\begin{tabular}{lcccc}
\hline Solvent & $\begin{array}{c}\text { Density } \\
\left(\mathrm{kg} \cdot \mathrm{m}^{-3}\right)\end{array}$ & $\begin{array}{l}\text { Viscosity } \\
\left(10^{-4} \mathrm{~Pa} \cdot \mathrm{s}\right)\end{array}$ & $\begin{array}{l}\text { Surface tension } \\
\left(10^{-2} \mathrm{~N} \cdot \mathrm{m}^{-1}\right)\end{array}$ & $\begin{array}{l}\text { Rate of evaporation } \\
\left(10^{-3} \mathrm{~g} \cdot \mathrm{min}^{-1} \cdot \mathrm{m}^{-2}\right)\end{array}$ \\
\hline Xylene & 840 & 7.5 & 3.0 & $1.1 \pm 0.5$ \\
\hline Solute & $\begin{array}{c}\text { Density } \\
\left(\mathrm{kg} \cdot \mathrm{m}^{-3}\right)\end{array}$ & $\begin{array}{l}\text { Diffusion coefficient in xylene } \\
\left(10^{-10} \mathrm{~m}^{2} \cdot \mathrm{s}^{-1}\right)\end{array}$ \\
\hline Polystyrene & 1050 & & 6.05 \\
\hline
\end{tabular}

of gravity and surface tension in spreading are measured by the Bond number Bo $=\rho g R_{0}^{2} / \gamma$, where $\rho$ is the density of the liquid, $g$ is the gravitational acceleration, $\gamma$ is the surface tension and $R_{0}$ is initial radius of droplet. When the initial radius is much less than $1.87 \mathrm{~mm}$, effect of gravity on the shape of the droplet can be ignored. In order to rule out the effects of gravity, a droplet with the volume of $0.7 \pm 0.015 \mu$ l whose radius is about $0.54 \pm 0.15$ $\mathrm{mm}$ is produced by microsyringe and deposited on the polystyrene surface using a micropipette. During the experiments, the temperature always maintains at $23^{\circ} \mathrm{C}$, and the pressure $101.3 \mathrm{kPa}$. In standard condition, we obtain the average rate of evaporation of xylene is $1.1 \times 10^{-3} \mathrm{~g} \cdot \mathrm{min}^{-1} \cdot \mathrm{m}^{-2}$ by experiments. The mass loss $\left(3 \times 10^{-9} \mathrm{~g} / \mathrm{min}\right)$ result from evaporation is much less than the total liquid mass $\left(5.46 \times 10^{-4} \mathrm{~g}\right)$. Therefore, the influence of xylene's evaporation to droplet is negligible. Other data about the properties of solute and solvent is listed in the Table 1. The experimental pictures are analyzed by software (SCA202 V.3.61.4, DataPhysics). The data of geometrical parameters is the average five experimental results.

\subsection{Verification test}

The relative influences of convection and diffusion in flow field are measured by the Pelect number $P e=v L / D$, where $v$ is characteristic velocity, $L$ is characteristic length, $D$ is the diffusion coefficient. In this case, $P e \sim 10^{-2}$. According to the Pelect number, we cannot ruled out the influence of convection completely. So we use a concise and district method to determine the influence of convection to mass transfer in our case, which compare the dissolution process of any point (point $A$ as an example) that near the origin and far away from the contact line (see Fig. 3a) with that of the verification test (see Fig. 1d).

The verification test is a 1D dissolution which can ignore the influence of wetting and convection. The test device is a thin glass tube, and the lower part of glass tube is filled with polystyrene, and the xylene is poured into tube from the upper part of glass
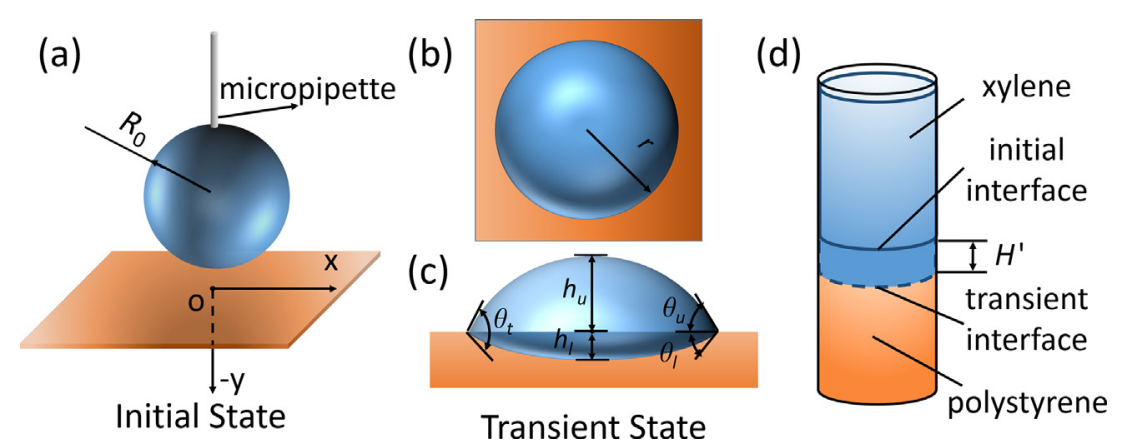

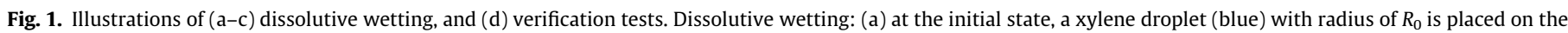

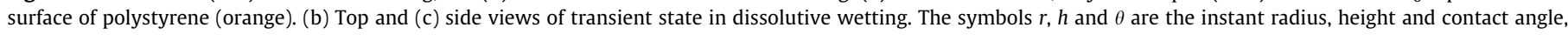

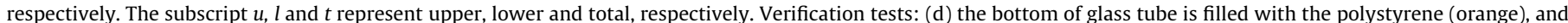

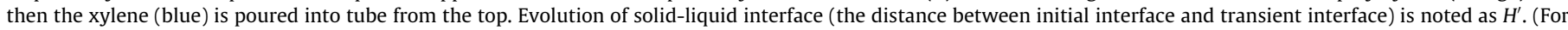
interpretation of the references to color in this figure legend, the reader is referred to the web version of this article.) 
tube. Then the microscope is used to observe and record the change of solid-liquid interface. The dissolution interface in the verification tests is nearly flat. In Fig. 3c, the black points are the data measured in the experiments. The result is shown in the Fig. $3 c$. In this case, if convection plays a key role in the mass transport, the diffusion speed will be much quicker than 1D dissolution. We can find that two sets of points In Fig. 3c, which are the results of $1 \mathrm{D}$ dissolution and the dissolution process of any point in substrate in dissolutive wetting, are distributed in the same zone in the diagram. Therefore, we think the convection effect on mass transfer is very small.

\section{Results}

The schematic of the dissolutive wetting problem is shown in Fig. 1a-c, a section in the $\mathrm{x}-\mathrm{y}$ plane of a droplet wetting a dissolvable substrate. The polystyrene begins to dissolve into droplet, when liquid contacts with substrate. The top and side views of dynamic process of the dissolutive wetting are shown in Fig. 2.
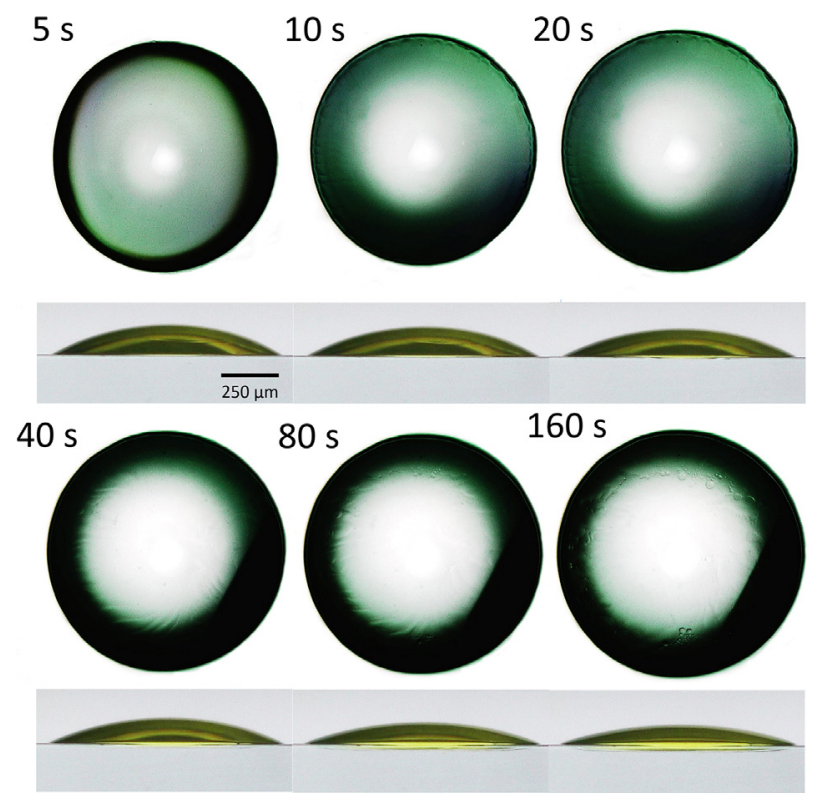

Fig. 2. A history of a dissolutive wetting experiment of xylene on polystyrene recorded using a microscope (KH-8700, Hirox). The droplet has $0.125 \%$ (mass ratio) perylenedicarboxylic acid bis (2-methylpropyl) ester and becomes green under the excitation of white light. The side view of experiment is taken in dark field, and then top view of droplet is taken by using Coaxial lens (AD-5040CS).

\subsection{Theoretical analysis}

In the process of dissolutive wetting, the mechanisms such as surface tension, viscosity and inertial exist simultaneously, and they influence the interfacial shape in different extent [30]. In order to clarify the relationship between different forces, a relevant dimensionless-number space should be established, which consists of Bond number, Reynolds number and Capillary number $[31,32]$. The relative influences of viscosity and surface tension are measured by the capillary number $\mathrm{Ca}=\mu \nu / \gamma$, where $\mu$ is the dynamic viscosity of the fluid, $\gamma$ is the surface tension, $v$ is characteristic velocity. For wetting in drop transfer, $\mathrm{Ca} \sim 10^{-6}$. The Reynolds number $R e=\rho v L / \mu$, where $L$ is characteristic length, measures the ratio of inertial forces to viscous forces. In this case, $R e \sim 10^{-7}$. During the process of dissolutive wetting, the influence of gravity can be ignored; while surface tension plays a main role, owing to $\mathrm{Bo} \ll 1$. It is rational to assume the shape of upper liquid is spherical cap. Considering the axial symmetric interface, the problem about interface evolution is three-dimension. Our goal is to find the deformation of the solid $h(x, t)$ firstly and then obtain the evolution of vapor-liquid interface according to the volume alteration of liquid.

\subsection{1. $1 D$ dissolution}

According to the results of verification test, the dissolution process of any point in droplet is nearly same as that of $1 \mathrm{D}$ dissolution. We take the influence of convection to droplet's shape can be ignored in our case. We can describe the dissolution base on Noyes-Whitney equation [33]

$\frac{d m}{d t_{d}}=A \frac{D}{d}\left(C_{s}-C\right)$

where $m$ is the dissolution mass, $t_{d}$ is the dissolution time, $A$ is the contact area, $D$ is the diffusion coefficient, $d$ is the diffusion layer thickness, $C_{s}$ is the mass concentration of the solute in the diffusion layer on the substrate surface, $C$ is mass concentration of the solute in droplet.

\subsubsection{The wetting of droplet}

The wetting process is self-similarity (see Fig. 4), so it can be expressed as $r / r_{0}=a\left(t_{0} / \tau_{0}\right)^{n}$ by scaling law, where $r_{0}$ is the radius of droplet, $a$ is the wetting coefficient, $\tau_{0}$ is the characteristic time of wetting, $t_{0}$ is the time consumed to reach $r$, and $n$ is the scaling exponent. According to the calculating results of fluid theory using lubrication approximation [36,37], the position of contact line is proportional to the one-tenth power law of the time. In our experiments, we find the scaling exponent of the dissolutive wetting is
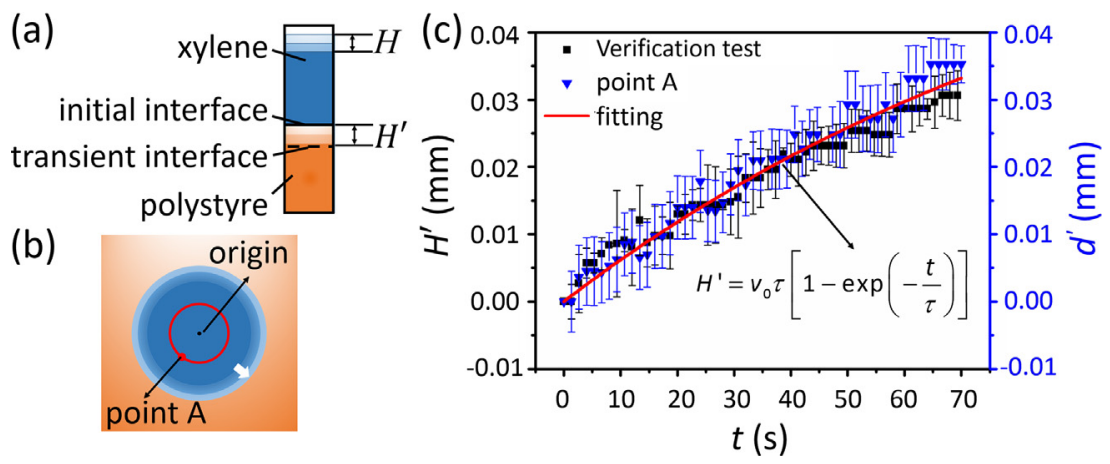

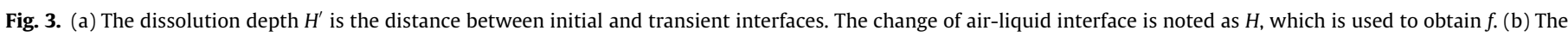

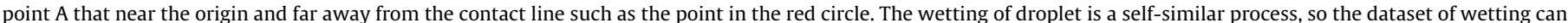

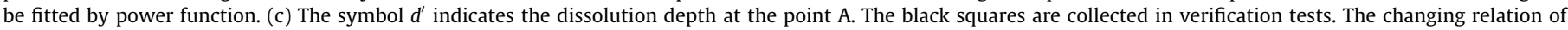
dissolution depth with time can be described by exponential function $H^{\prime}=v_{0} \tau[1-\exp (-t / \tau)]$, giving $v_{0}=6.5 \times 10^{-4} \mathrm{~mm} / \mathrm{s}$ and $\tau=105 \mathrm{~s}$. 


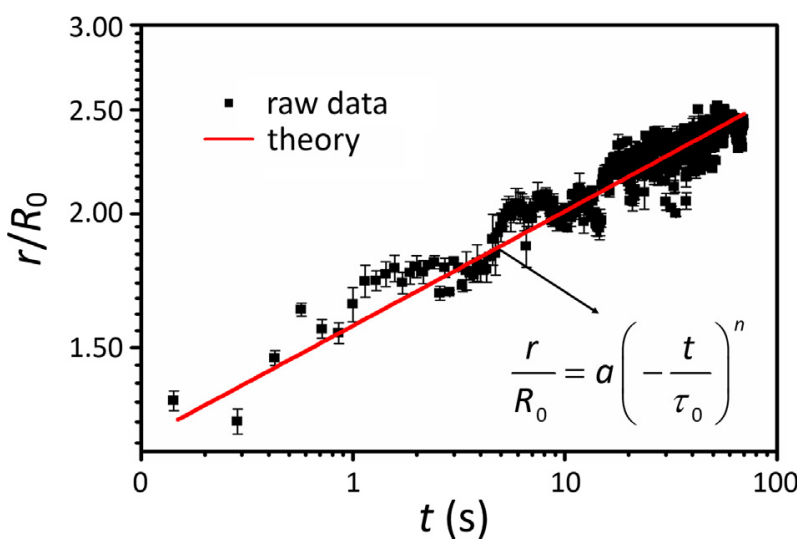

Fig. 4. The log-log plot reveals a power-law relation between time $t$ and wetting radius $r$, with a scaling exponent $n=0.11$. Other relevant parameters are characteristic time of wetting $\tau_{0}=40 \mathrm{~s}$, initial radius $r_{0}=0.54 \mathrm{~mm}$ and the wetting coefficient $a=2.25$, respectively.

0.11 (see Fig. 3c). This exponent is greater than Tanner's law [38] for surface tension driven spreading. There is a difference between these two power laws because the dissolution promotes the wetting of droplet.

\subsubsection{The couple of dissolution and wetting}

The substrate dissolves into solvent as soon as the droplet contacts with it. The starting time at which each point on the substrate begins to dissolve is determined by wetting. Therefore, we can obtain the revolution of solid-liquid interface by combining Noyes-Whitney equation and wetting equation

$h(x, t)=-v_{0} \tau\left[1-\exp \left(\frac{\tau_{0}}{\tau}\left(\frac{x}{a r_{0}}\right)^{\frac{1}{n}}-\frac{t}{\tau}\right)\right]$,

where $t$ is the time of dissolutive wetting. The dissolution depth of origin is given by the function $h_{l}(t)=v_{0} \tau[1-\exp (-t / \tau)]$. Finally, the upper cap's shape of droplet can be obtained by considering the change of liquid volume. The upper part of droplet is spherical cap, and the volume of droplet satisfies the $V_{l}(t)=V_{0}+f V_{s}(t)=$ $V_{u}+V_{s}(t)$, where $V_{0}$ is the initial volume of droplet, $V_{s}$ is dissolution volume of solid, $V_{u}$ is the volume of upper liquid cap, and $f$ is the ratio of increased liquid volume to dissolved solid volume. The value of $f$ can be obtained by measuring the change of solvent volume in verification test (see Fig. 1d). Fig. 5 shows both the experimental and theoretical results of interfacial shape.

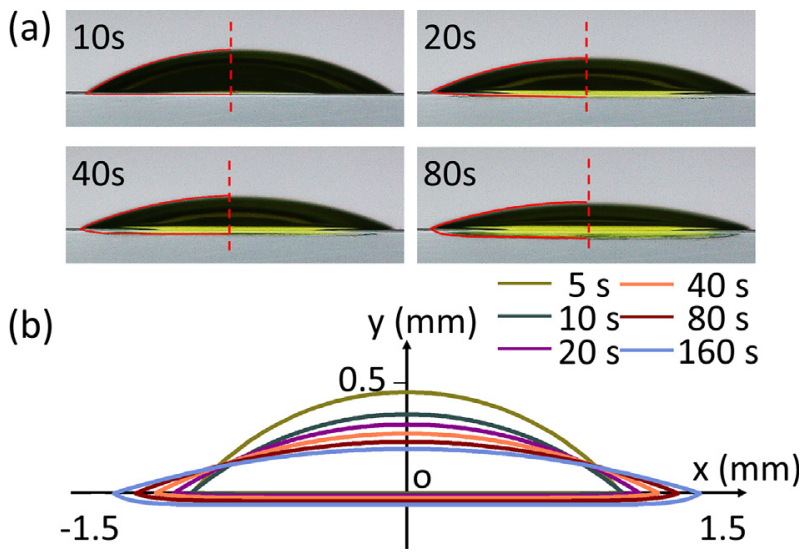

Fig. 5. Evolution of the interfacial shape. (a) The theoretical interfaces (red line) are compared with the experimental results. (b) The theoretical evolution of interfacial shape is expressed by different colors. (For interpretation of the references to color in this figure legend, the reader is referred to the web version of this article.)
Dissolutive wetting involves both dissolution and wetting. Now that convection could be ignored in our cases from the verification tests, diffusion of solute in solvent can be describe by diffusion equation. Coupled with Eq. (2), we obtain distribution of transient mass concentration $c$ in droplet

$c=C_{s}\left[1-\operatorname{erf}\left(\frac{Y}{2 \sqrt{D t^{\prime}}}\right)\right]$

where $Y=y-h_{l}+h, t^{\prime}=t-\tau_{0}(x / a R)^{1 / n}, D$ is the diffusion coefficient. The results of mass concentration in droplet are shown in Fig. 6.

Through fitting the wetting equation to the dataset of wetting (see Fig. 3e), we can obtain other parameters. The characteristic time of wetting $\tau_{0}=40 \mathrm{~s}$, and the wetting coefficient $a=2.25$. The corresponding dissolution parameters are given by fitting the curve Fig. 3a to the dataset of verification tests. The initial dissolution velocity $v_{0}=6.5 \times 10^{-4} \mathrm{~mm} \cdot \mathrm{s}^{-1}$, and the characteristic time of dissolution $\tau=105 \mathrm{~s}$. The ratio of increased liquid volume to dissolved solid volume $f$ is 0.9 . The mass concentration of the solute in the diffusion layer on the substrate surface $C_{S}$ is 0.41 . The diffusion coefficient $D$ is chosen to be $6.05 \times 10^{-4} \mathrm{~mm}^{2} \cdot \mathrm{s}^{-1}$, according to the literature $[34,35]$.

\subsection{Evolution of geometry parameters}

Fig. $7 c-d$ (the solid line and point show the theoretical and experimental results of the variation of key geometry parameters with time respectively) display that the upper contact angle fall sharply then decreases slowly. The shape of upper cap especially the upper contact angle change sharply in a short time. The upper height of droplet also has plunging. On the other hand, the lower angle keeps almost linearly increasing indicates that change tendency of lower contact angle is determined by a factor that is not time-sensitive. According to the analysis results of the model, the wetting factor $n$ determines the trend in lower contact angle. Considering the results of contact angle, the total contact angle will increase gradually after falling (see Fig. 8b). In Fig. 7, the value of lower height and lower contact angle is zero within the first five seconds because the lower height is too small to be distinguished by the camera.

\subsection{The two stages in dissolutive wetting}

By calculating the speed ratio of wetting to dissolution (see Fig. 8c), we find that change of interface has two stages. Here, we use $\bar{v}$ to denote the speed ratio of wetting to dissolution. In the first stage, $\bar{v}>10$, which means that surface tension is a main driving force, compare with dissolution. Change of contact angle depends mainly on the wetting of droplet, and the lower interface of droplet near the origin begins to dissolve almost at the same time. In this stage, the velocity of wetting is far faster than that of dissolution for droplet. For the zone near the origin, the difference in dissolution time can be ignored. Moreover, we can find that the concentration gradients around the origin are similar in Fig. 6. Therefore, the lower interface near the origin has small curvature.

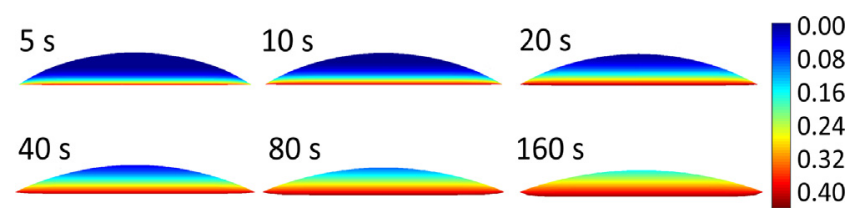

Fig. 6. Distribution and evolution of concentration in droplet. In the situation of ignoring convection, the Eq. (3) can be used to calculate the evolution of concentration in droplet. 

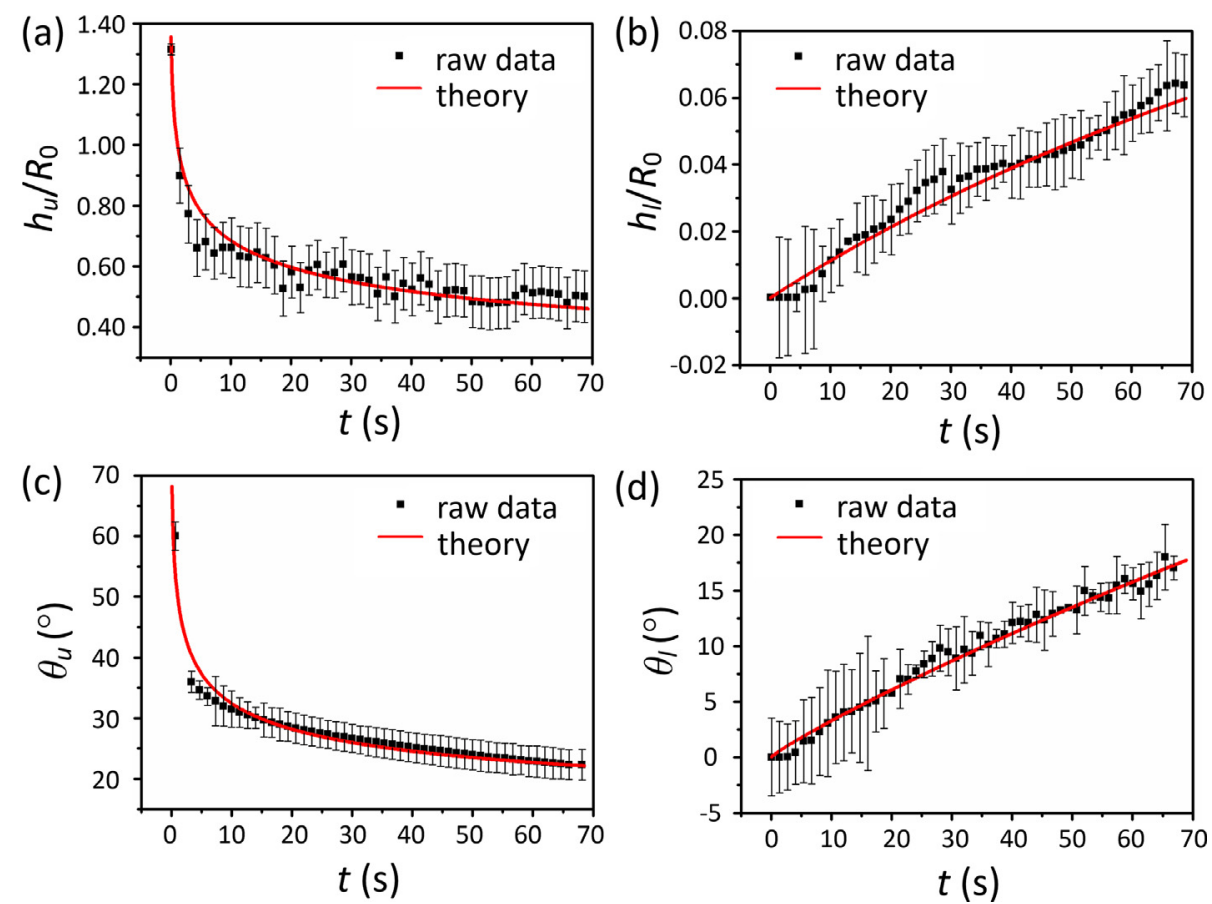

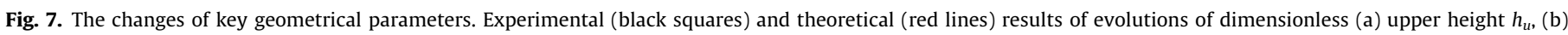

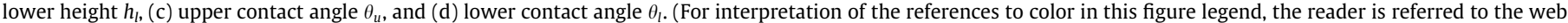
version of this article.)
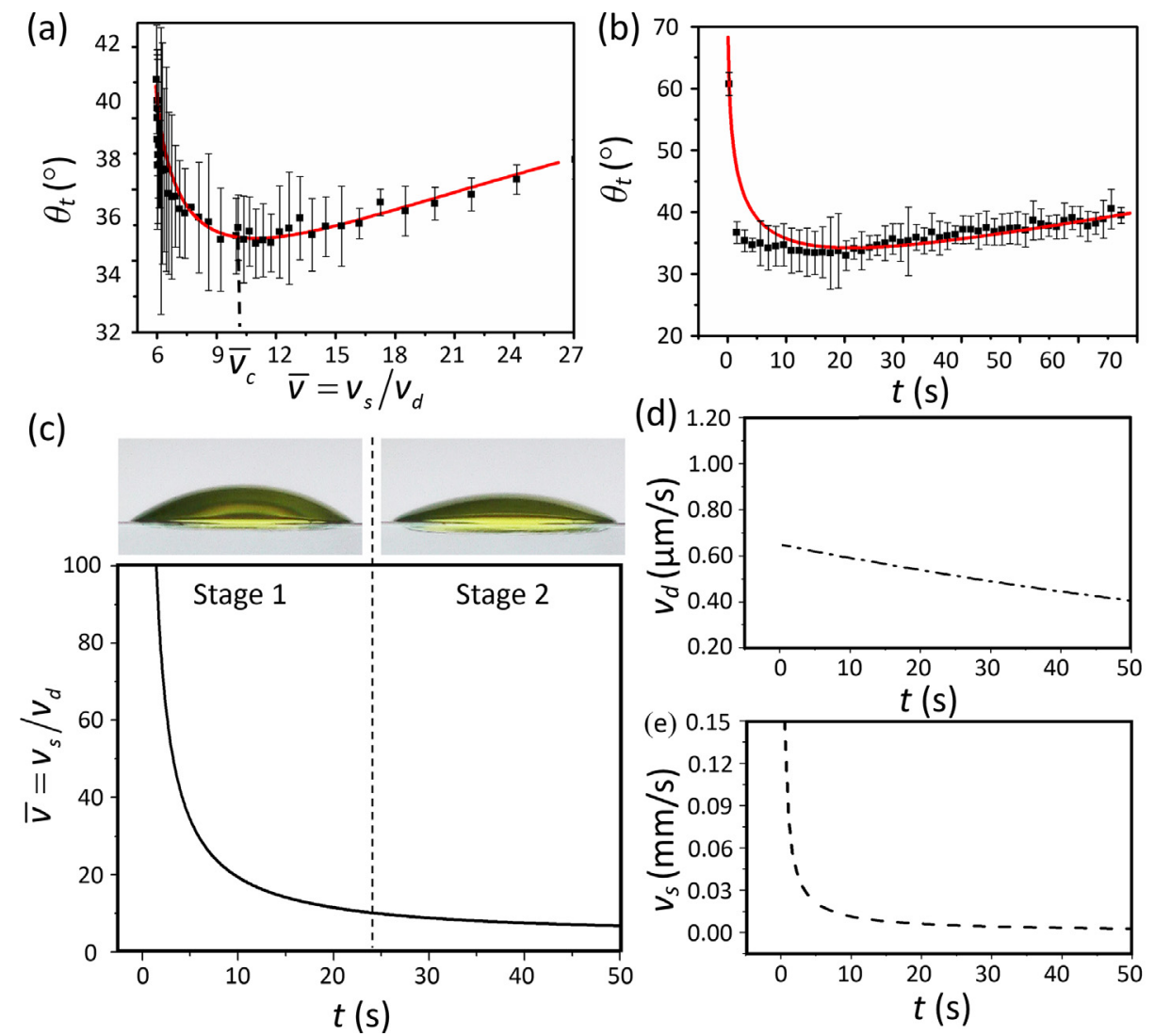

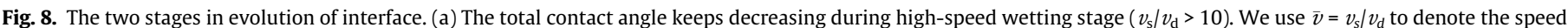

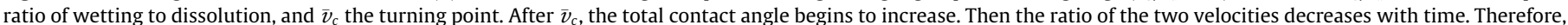

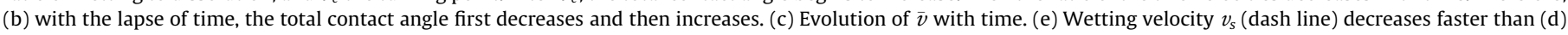
dissolution velocity $v_{d}$ (dash-dot line). The turning point $\bar{v}_{c}$ is at time of about $24 \mathrm{~s}$. 
On the other hand, the decreasing velocity of upper contact angle is quicker than the increasing of lower contact angle. Therefore, total contact angle decreases in this stage (see Fig. 8a). In the second stage, the speed of wetting slows down to the same order of dissolution speed; change of interface depends on coupling of wetting and dissolution. The data shown in Fig. 8a also verifies the existence of two stages. After the turning point, the slow wetting curbed the decrease of upper angle, while the lower contact angle keeps rising. Therefore, the total contact angle will increase gradually in this stage. In addition, the increase in contact angle is proportional to time after $24 \mathrm{~s}$ (see Fig. $8 \mathrm{~b}$ ), because the speed ratio of wetting to dissolution is relative stable in the second stage.

\section{Discussion and conclusions}

In summary, we carry out experiments to record revolution of interfacial shape in the dissolutive wetting. We measure changes of geometry parameter, and reveal scaling exponent of the dissolutive wetting is 0.11 . This scaling exponent is agree with the conclusion about wetting scaling exponent in the literature [31]. The dissolution kinetic will increase the wetting velocity [39]. We propose a model to describe the evolution of the interfacial shape and droplet concentration. The theoretical interface shape is similar with the experimental results in literature [31]. In this way, we can understand the mechanisms which effect change of interface in dissolutive wetting. Our results show that the process of dissolutive wetting exists two stages, and the ratio of speed plays a key role in interface evolution. In the first stage, the wetting effect is dominant, and the evolution of boundary is controlled by wetting before the turning point. In the central of dissolution hole, the solid-liquid interface is flat because wetting speed is much faster than dissolution speed in this stage. With the decrease of wetting velocity, the dissolutive wetting enters the second stage. In this stage, the order of wetting speed is the same as that of dissolution speed, so wetting and dissolution mechanisms lead the variation of interfacial shape together. The deformation speed of upper cap slows down, and the total contact angle keeps increase linearly. With the transformation of droplet, the lower contact angle will over the upper contact angle. As for the increasing of lower contact angle, we should note that the trend of lower contact angle is decided by scaling exponent $n$. The second stage lasts relatively long and is the main component of dissolutive wetting. Protsenko et al. [28] also found the two stages of dissolutive wetting in the experiment of $\mathrm{Si}-\mathrm{Cu}$ system. Our studies demonstrate that the speed ratio of wetting to dissolution $\bar{v}$ is an important parameter for reflecting the coupling of wetting and dissolution in dissolutive wetting. In addition, the model developed here has applications in the fields such as MEMS and medicine, as an approach to predict and control evolution of interfacial shape caused by dissolutive wetting, as well as to improve protection of key components of equipment.

\section{Conflict of interest}

The authors declared that there is no conflict of interest.

\section{Acknowledgements}

This work was jointly supported by the National Natural Science Foundation of China (NSFC, Grant Nos. 11722223, 11672300, 11611130166 and U1562105), Royal Society-Newton Mobility Grant (Ref. IE150674), the CAS Key Research Program of Frontier Sciences (Grant No. QYZDJ-SSW-JSC019), the Strategic Priority Research Program of the Chinese Academy of Sciences
(Grant No. XDB22040401), and Young Elite Scientists Sponsorship Program by CAST (Grant No. 2015QNRC001).

\section{References}

[1] K. Landry, N. Eustathopoulos, Dynamics of wetting in reactive metal/ceramic systems: linear spreading, Acta Mater. 44 (1996) 3923-3932.

[2] A. Passerone, M.L. Muolo, F. Valenza, F. Monteverde, N. Sobczak, Wetting and interfacial phenomena in Ni-HfB2 systems, Acta Mater. 57 (2008) 356-364.

[3] R. Hellmann, S. Cotte, E. Cadel, S. Malladi, L.S. Karlsson, S. Lozano-Perez, M. Cabié, A. Seyeux, Nanometre-scale evidence for interfacial dissolutionreprecipitation control of silicate glass corrosion, Nat. Mater. 14 (2015) $307-$ 311.

[4] S.D. Mithani, V. Bakatselou, C.N. TenHoor, J.B. Dressman, Estimation of the increase in solubility of drugs as a function of bile salt concentration, Pharm. Res. 13 (1996) 163-167.

[5] K. Swaminathan, M.V. Panchagnula, Spreading and hole formation in natural oil films on aqueous solutions, Colloids Surf., A 520 (2017) 796-804.

[6] J. Schneider, P. Rohner, P. Galliker, S.N. Raja, Y. Pan, M.K. Tiwari, D. Poulikakos, Site-specific deposition of single gold nanoparticles by individual growth in electrohydrodynamically-printed attoliter droplet reactors, Nanoscale 7 (2015) 9510-9519.

[7] C. Wang, S.W. Nam, J.M. Cotte, C.V. Jahnes, E.G. Colgan, R.L. Bruce, M. Brink, M F. Lofaro, J.V. Patel, L.M. Gignac, E.A. Joseph, S.P. Rao, G. Stolovitzky, S. Polonsky, Q. Lin, Wafer-scale integration of sacrificial nanofluidic chips for detecting and manipulating single DNA molecules, Nat. Commun. 8 (2017) 14243.

[8] H. Wang, L.A. Zepeda-Ruiz, G.H. Gilmer, M. Upmanyu, Atomistics of vapourliquid-solid nanowire growth, Nat. Commun. 4 (2013) 1956.

[9] S. Biswas, J. Doherty, D. Saladukha, Q. Ramasse, D. Majumdar, M. Upmanyu, A Singha, T. Ochalski, M.A. Morris, J.D. Holmes, Non-equilibrium induction of tin in germanium: towards direct bandgap Ge1-xSnx nanowires, Nat. Commun. 7 (2016) 11405.

[10] C.W. MacMinn, J.A. Neufeld, M.A. Hesse, H.E. Huppert, Spreading and convective dissolution of carbon dioxide in vertically confined, horizontal aquifers, Water Resour. Res. 48 (2012) 12286.

[11] T. Geisler, A. Janssen, D. Scheiter, T. Stephan, J. Berndt, A. Putnis, Aqueous corrosion of borosilicate glass under acidic conditions: a new corrosion mechanism, J. Non-Cryst. Solids 356 (2010) 1458-1465.

[12] C.Y. Chen, C.P. Wong, Unveiling the shape-diversified silicon nanowires made by HF/HNO3 isotropic etching with the assistance of silver, Nanoscale 7 (2015) 1216-1223.

[13] B. Lang, K.T. Chow, R.O. Williams, Thin film freezing-template emulsion of itraconazole to improve the dissolution properties of poorly water-soluble drugs, J. Drug Del. Sci. Technol. 24 (2014) 205-211.

[14] D.R. Lee, M.J. Ho, H.J. Jung, H.R. Cho, J.S. Park, S.H. Yoon, Y.S. Choi, Y.W. Choi, C. H. Oh, M.J. Kang, Enhanced dissolution and oral absorption of tacrolimus by supersaturable self-emulsifying drug delivery system, Int. J. Nanomed. 11 (2016) 1109-1117.

[15] A. Arya, A. Chandra, V. Sharma, K. Pathak, Fast dissolving oral films: an innovative drug delivery system and dosage form, Int. J. Chem. Tech. Res. 2 (2010) 576-583.

[16] D. Desai, B. Wong, Y. Huang, Q. Ye, D. Tang, H. Guo, M. Huang, P. Timmins Surfactant-mediated dissolution of metformin hydrochloride tablets: wetting effects versus ion pairs diffusivity, J. Pharm. Sci. 103 (2014) 920-926.

[17] Y. Sui, H. Ding, P.D.M. Spelt, Numerical Simulations of Flows with Moving Contact Lines, Annu. Rev. Fluid Mech. 46 (2014) 97-119.

[18] J. Zhang, M.K. Borg, J.M. Reese, Multiscale simulation of dynamic wetting, Int. J. Heat Mass Transf. 115 (2017) 886-896.

[19] Q. Yuan, Y.P. Zhao, Precursor film in dynamic wetting, electrowetting, and electro-elasto-capillarity, Phys. Rev. Lett. 104 (2010) 246101.

[20] F. Yost, E. O'Toole, Metastable and equilibrium wetting states in the Bi-Sn system, Acta Mater. 46 (1998) 5143-5151.

[21] J.A. Warren, W. Boettinger, A. Roosen, Modeling reactive wetting, Acta Mater 46 (1998) 3247-3264.

[22] R.J. Braun, B.T. Murray, W.J. Boettinger, G.B. McFadden, Lubrication theory for reactive spreading of a thin drop, Phys. Fluids 7 (1995) 1797-1810.

[23] N. Alleborn, H. Raszillier, Spreading and sorption of a droplet on a porous substrate, Chem. Eng. Sci. 59 (2004) 2071-2088.

[24] W. Villanueva, K. Grönhagen, G. Amberg, J. Ågren, Multicomponent and multiphase modeling and simulation of reactive wetting, Phys. Rev. E 77 (2008) 056313.

[25] E.B. Webb, G.S. Grest, D.R. Heine, J. Hoyt, Dissolutive wetting of Ag on Cu: a molecular dynamics simulation study, Acta Mater. 53 (2005) 3163-3177.

[26] G.C. Mo, W.Y. Liu, D.Y. Kwok, Surface-ascension of discrete liquid drops via experimental reactive wetting and lattice Boltzmann simulation, Langmuir 21 (2005) 5777-5782.

[27] S. Su, The Development of Computational Models for Studying Wetting, Evaporation and Thermal Transport for Electronics Packaging Applications, PhD Dissertation, Dept Mech Eng, SUNY, Binghamton, 2012.

[28] P. Protsenko, O. Kozlova, R. Voytovych, N. Eustathopoulos, Dissolutive wetting of Si by molten Cu, J. Mater. Sci. 43 (2008) 5669-5671.

[29] L. Yin, B.T. Murray, T.J. Singler, Dissolutive wetting in the Bi-Sn system, Acta Mater. 54 (2006) 3561-3574.

[30] Q. Yuan, Y.-P. Zhao, Statics and dynamics of electrowetting on pillar-arrayed surfaces at the nanoscale, Nanoscale 7 (2015) 2561-2567. 
[31] T.J. Singler, S. Su, L. Yin, B.T. Murray, Modeling and experiments in dissolutive wetting: a review, J. Mater. Sci. 47 (2012) 8261-8274.

[32] J. Chapuis, E. Romero, F. Soulié, C. Bordreuil, G. Fras, Transient behaviour of deposition of liquid metal droplets on a solid substrate, Heat Mass Transf. 52 (2015) 2283-2292.

[33] A. Noyes, W.R. Whitney, Drug dissolution, J. Am. Chem. Soc. 19 (1897) 930934.

[34] J.M. Zielinski, J. Duda, Predicting polymer/solvent diffusion coefficients using free-volume theory, AIChE J. 38 (1992) 405-415.

[35] Y. Iwai, M. Kohno, T. Akiyama, Y. Arai, Measurement and correlation of mutual diffusion coefficients for molten polystyrene-hydrocarbon systems, Polym. Eng. Sci. 27 (1987) 837-841.
[36] L.W. Schwartz, R.R. Eley, Simulation of droplet motion on low-energy and heterogeneous surfaces, J. Colloid Interface Sci. 202 (1998) 173-188.

[37] G. Barenblatt, E. Beretta, M. Bertsch, The problem of the spreading of a liquid film along a solid surface: A new mathematical formulation, Proc. Natl. Acad. Sci. 94 (1997) 10024-10030.

[38] L. Tanner, The spreading of silicone oil drops on horizontal surfaces, J. Phys. D Appl. Phys. 12 (1979) 1473.

[39] Q.Z. Yuan, J.H. Yang, Y. Sui, Y.P. Zhao, Dynamics of dissolutive wetting: a molecular dynamics study, Langmuir 33 (26) (2017) 6464-6470. 